\title{
Follow-up study of 22 Chinese children with Alexander disease and analysis of parental origin of de novo GFAP mutations
}

\author{
Lili Zang ${ }^{1}$, Jingmin Wang ${ }^{1}$, Yuwu Jiang ${ }^{1}$, Qiang $\mathrm{Gu}^{1}$, Zhijie Gao ${ }^{1}$, Yanling Yang${ }^{1}$, Jiangxi Xiao ${ }^{2}$ and Ye $\mathrm{Wu}^{1}$
}

To delineate the phenotype and genotype in Chinese children with type I Alexander disease (AxD) and the parental origin of de novo glial fibrillary acidic protein (GFAP) mutations. Twenty-two children with clinically diagnosed type I AxD were followed up for 1.66-6.62 years. Allele-specific PCR was used for the analysis of parental origin of the allele harboring the de novo mutation. Phenotype of these patients were consistent with type I AxD described in other population, with developmental delay (motor delay in $81.82 \%$, cognitive delay in $63.64 \%$ ), macrocephaly $(100 \%)$, seizures $(95.45 \%$ ), paroxysmal deterioration $(27.27 \%)$ and typical brain magnetic resonance imaging (100\%). Progression was slower than reported. At 8.55 years of age (5.29-13.25), all patients who underwent the second follow-up were alive. Eleven heterozygous missense mutations of GFAP were identified in 21 patients, with three novel mutations. Reported hot spot mutations, p.R79, p.R239 and p.R88, were also identified in Chinese patients. Mutations were de novo in all but one case. The mother of a proband was demonstrated to be a presymptomatic patient with type II AxD with a p.R79H mutation. Ninety percent of de novo mutations were on the paternal allele demonstrated by allele-specific PCR. This is the largest follow-up study on Chinese children with AxD. The phenotypes of these patients are consistent with reports in other populations. GFAP mutations were identified in $95.46 \%$ of Chinese children with clinically diagnosed type I AxD. Our data suggested a male germ-line transmission.

Journal of Human Genetics (2013) 58, 183-188; doi:10.1038/jhg.2012.152; published online 31 January 2013

Keywords: Alexander disease; Chinese; follow-up study; GFAP; parental origin

\section{INTRODUCTION}

Alexander disease $(\mathrm{AxD})$ is a rare progressive leukoencephalopathy inherited in an autosomal dominant manner. Age-dependent clinical subtypes have been defined for almost 30 years, including infantile (onset before 2 years of age), juvenile (2-14 years) and adult AxD (late juvenile to adult). ${ }^{1}$ Overlaps between age-dependent subtypes, especially between juvenile and adult $\mathrm{AxD}$, have been described. ${ }^{2}$ Revised clinical subtypes defined as type I and II were proposed in 2011 based on a careful review of $215 \mathrm{AxD}$ cases. ${ }^{3}$ Type I is characterized by early onset (usually $<4$ years of age), macrocephaly, seizures, developmental delay, paroxysmal deterioration and typical magnetic resonance imaging (MRI) findings. Type II is characterized by later onset, autonomic dysfunction, ocular movement abnormalities, bulbar symptoms and atypical MRI findings. The pathological hallmark of $\mathrm{AxD}$ is the accumulation of Rosenthal fibers in astrocytes, composed of glial fibrillary acidic protein (GFAP), heat shock protein 27 and $\alpha \mathrm{B}$-crystallin., ${ }^{4,5}$ Diagnosis was on the basis of the neuropathological features before the identification of GFAP mutations in 2001. ${ }^{6}$ Nearly all $(>90 \%)$ cases with AxD harbor heterozygous GFAP mutations, ${ }^{7}$ and most of these mutations occur de novo. ${ }^{8}$ In this study, we performed a 1.66-6.62 year follow-up of 22
Chinese children with type I $\mathrm{AxD}$ and analyzed the parental origin of the allele bearing de novo mutations.

\section{PATIENTS AND METHODS}

Clinical diagnosis, assessment and follow-up

Clinical diagnosis of AxD was made on the basis of typical MRI features proposed by van der Knaap. ${ }^{9}$ We enrolled patients since 2005. In December 2010 and February 2012, follow-up was performed.

Motor function assessment was based on the Gross Motor Function Classification System (GMFCS), Chinese version. ${ }^{10}$ Levels I-V was classified according to the motor function and age of the children. For patients who had acquired the skill of walking, level I was classified as walking independently indoors and outdoors, level II as walking independently but with limitations on uneven surfaces and inclines, level III as walking with assistance, level IV as ambulance using a power wheelchair and level $\mathrm{V}$ as loss of independent mobility.

Gesell or Wechsler Intelligence Scale for Children was used for assessment of the cognitive function. The scale for cognitive function was classified as $\mathrm{C} 0$ (normal, IQ (intelligence quotient)/DQ (development quotient) $\geqslant 70$ ), C1 (mild impairment, IQ/DQ 55-69), C2 (moderate impairment, IQ/DQ 40-54) and $\mathrm{C} 3$ (severe impairment, $\mathrm{IQ} / \mathrm{DQ}<40$ ). 
Standard protocol approval and patient consent

The study was approved by the clinical research ethics committee of Peking University First Hospital, and informed consent was obtained from all parents.

\section{GFAP gene sequencing}

Genomic DNA was extracted from the white blood cells. The sequence of GFAP was obtained from the UCSC Genome Bioinformatics database (NM_002055). Nine exons and flanking introns were sequenced. Novel mutations found in patients were tested in 100 controls.

\section{Analyzing the parental origin of de novo GFAP mutations}

To determine the parental origin of the chromosome bearing the de novo mutation, allele-specific PCR was performed in 10 cases (Table 1). For each mutant nucleotide, informative single-nucleotide polymorphisms within $2 \mathrm{~kb}$ region flanking the mutation were selected. Subsequently, with one of the primer pair ending with the alternative nucleotide of the informative singlenucleotide polymorphism (for example, rs139617907, A or T), and a common primer, allele-specific PCR was performed to amplify the single-allele containing either the wild-type or mutant site. The parental origin of the mutation was determined based on the presence of the informative single-nucleotide polymorphism within the specifically amplified mutant allele. Each amplification was repeated for three times.

\section{RESULTS}

Twenty-two patients were clinically diagnosed with type I AxD, including 13 boys and 9 girls. All individuals were unrelated and children of non-consanguineous parents.

\section{Phenotype of patients}

Disease onset and signs at first visit. The developmental milestones of the motor function were slow in $81.82 \%(n=22)$ in the first year of life, with cognitive developmental delay in $63.64 \%$. At the first visit, the average age was 4.83 years $(0.54-12.08)$. The chief complaints were developmental delay in 5(22.73\%), recurrent seizures in 14 $(63.64 \%)$ or acute onset of walking disability after a falling in $3(13.64 \%)$. Macrocephaly was noticed in most patients, with head circumference of $44.00-58.00 \mathrm{~cm}, 6.33 \%(1.32-13.95 \%)$ above average. Physical examination showed hypertonia in $7(31.82 \%, n=21)$, hypotonia in 2 (9.52\%), hyperreflexia in 12 (57.14\%) and Babinski sign in $10(47.62 \%)$. At the first visit, GMFCS I was scored in 13 $(59.09 \%, n=22)$, II in $5(22.73 \%)$, IV in $1(4.54 \%)$, and $\mathrm{V}$ in 3 $(13.64 \%)$. Eight cases $(36.36 \%, n=22)$ showed no cognitive impairment. Mild cognitive dysfunction (C1) was found in $8(36.36 \%)$, C2 in $2(9.10 \%)$, and C3 in $4(18.18 \%)$. Brain MRI was performed at an age of 0.67-6.00 years (Figure 1). Blood biochemistry and metabolic test were unremarkable in all cases. Symptoms and sign at first visit were shown in Table 2.

Disease progression. $0.13-5.41$ years after the first visit, 19 patients underwent the first follow-up, with an average age of 7.34 years (1.3814.71). 1.66-6.62 years after the first visit, 14 underwent the second follow-up, with the average age 8.55 years (5.29-13.25). Some cases were not followed because of failure to make contact.

Progression of motor and cognitive impairment. At the first follow-up, motor function was classified as GMFCS I in $10(52.63 \%, n=19)$, II in $6(31.58 \%)$, IV in $1(5.26 \%)$ and V in $2(10.53 \%)$. Compared with the first visit, $14(73.68 \%)$ remained stable, 3 (15.79\%) showed regression and $2(10.53 \%)$ showed improvement. Most patients $(84.21 \%)$ preserved the ability to walk independently. At the second follow-up 1.25 years later, $6(42.86 \%, n=14)$ were classified as GMFCS I, $3(21.43 \%)$ as II, $2(14.29 \%)$ as III and $3(21.43 \%)$ as IV. Compared with the first follow-up, $10(71.43 \%)$ remained stable, while $4(28.57 \%)$ showed regression and could not walk by themselves.

Table1 Primers and results of allele-specific PCR

\begin{tabular}{|c|c|c|c|c|c|c|}
\hline \multirow[b]{2}{*}{ Case } & \multirow[b]{2}{*}{ Mutation } & \multirow{2}{*}{$\begin{array}{l}\text { Single-nucleotide } \\
\text { polymorphism }\end{array}$} & \multicolumn{2}{|c|}{ Allele-specific PCR primers } & \multirow[b]{2}{*}{ Products size (bp) } & \multirow{2}{*}{$\begin{array}{c}\text { Paternal/Maternal } \\
\text { allele bearing the } \\
\text { mutation }\end{array}$} \\
\hline & & & Forward primer $\left(5^{\prime} \rightarrow 3^{\prime}\right)$ & Reverse primer $\left(5^{\prime} \rightarrow 3^{\prime}\right)$ & & \\
\hline \multirow[t]{2}{*}{3} & $\mathrm{R} 88 \mathrm{C}$ & rs111979924 & 5'-CTCTGGGCACAGTGACCTC-3' & 5'-AGGCTCAGAATAGGTGAGCTCG-3' & 977 & M \\
\hline & & & & 5'-AGGCTCAGAATAGGTGAGCTCA-3' & & \\
\hline \multirow[t]{2}{*}{4} & R239C & rs78435425 & 5'-CTTGTGACCCCCATCAAGTT-3' & 5'-ATTGCCTCATACTGCGTGCG-3' & 1240 & $P$ \\
\hline & & & & 5'-ATTGCCTCATACTGCGTGC $\boldsymbol{A}$-3' & & \\
\hline \multirow[t]{2}{*}{5} & R239C & rs139617907 & 5'-AGAGACACTCAGAGAGAGGAGAG $\boldsymbol{A}-3^{\prime}$ & 5'-TATTCTCCCAGCTTCCTCCA-3' & 470 & $P$ \\
\hline & & & 5'-AGAGACACTCAGAGAGAGGAGAG $T-3^{\prime}$ & & & \\
\hline \multirow[t]{2}{*}{8} & R79L & rs111979924 & 5'-CTCTGGGCACAGTGACCTC-3' & 5'-AGGCTCAGAATAGGTGAGCTCG-3' & 977 & $P$ \\
\hline & & & & 5'-AGGCTCAGAATAGGTGAGCTCA-3' & & \\
\hline \multirow[t]{2}{*}{10} & R88S & rs2070935 & 5'-TAGAGAGAGGGTCCTCTTGA-3' & 5'-CACTCCTTCTTGGGGATTCA-3' & 808 & $P$ \\
\hline & & & 5'-TAGAGAGAGGGTCCTCTTG $\boldsymbol{C}$ - $3^{\prime}$ & & & \\
\hline \multirow[t]{2}{*}{15} & N77K & rs28485918 & 5'-CCTATCAGGACCTCCACTGC-3' & 5'-TGTAGCTGGCAAAGCGGTCA-3' & 1110 & $P$ \\
\hline & & & & 5'-TGTAGCTGGCAAAGCGGTC $T$-3' & & \\
\hline \multirow[t]{2}{*}{18} & $\mathrm{R} 239 \mathrm{H}$ & rs111979924 & 5'-TGAATCCCCAAGAAGGAGTG-3' & 5'-CATTGCCTCATACTGCGTG $\boldsymbol{C}$-3' & 1668 & $P$ \\
\hline & & & & 5'-CATTGCCTCATACTGCGTG $T$-3' & & \\
\hline \multirow[t]{2}{*}{19} & $\mathrm{R} 79 \mathrm{C}$ & rs111979924 & $5^{\prime}$-GATGATGGAGCTCAATGAC $c-3^{\prime}$ & 5'-CCTCTGATCCCAGGTAACC $A-3^{\prime}$ & 811 & $P$ \\
\hline & & & 5'-GATGATGGAGCTCAATGAC $T$-3' & & & \\
\hline \multirow[t]{2}{*}{20} & $\mathrm{R} 239 \mathrm{H}$ & rs111979924 & 5'-TGAATCCCCAAGAAGGAGTG-3' & 5'-CATTGCCTCATACTGCGTGC-3' & 1668 & $P$ \\
\hline & & & & 5'-CATTGCCTCATACTGCGTG $T$-3' & & \\
\hline \multirow[t]{2}{*}{21} & $\mathrm{R} 239 \mathrm{H}$ & rs111979924 & 5'-TGAATCCCCAAGAAGGAGTG-3' & 5'-CATTGCCTCATACTGCGTGC-3' & 1668 & $P$ \\
\hline & & & & 5'-CATTGCCTCATACTGCGTG $T$-3' & & \\
\hline
\end{tabular}

Abbreviations: M, maternal; $\mathrm{P}$, paternal; The $3^{\prime}$ ending of the specific primers are shown in bold and italic. 

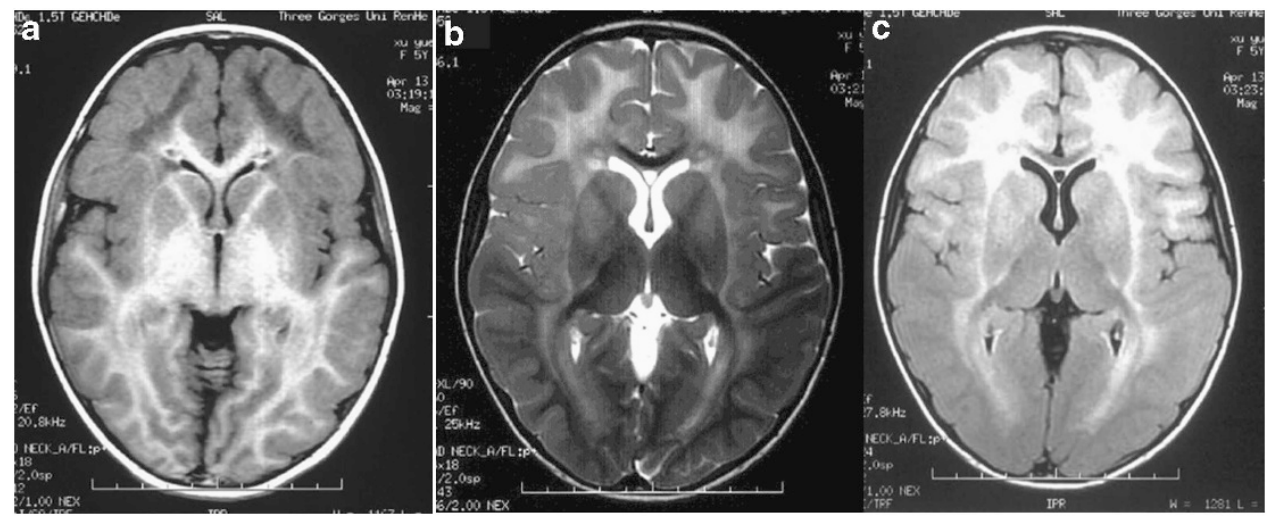

Figure 1 Typical brain magnetic resonance imaging in one of our patients with type I AxD. (a) (T1 image), (b) (T2 image) and (c) (Falir image) illustrate the characteristic abnormalities in white matter with predominance in the frontal lobe, and swelling of the basal ganglia.

Table 2 Clinical features of 22 AxD patients

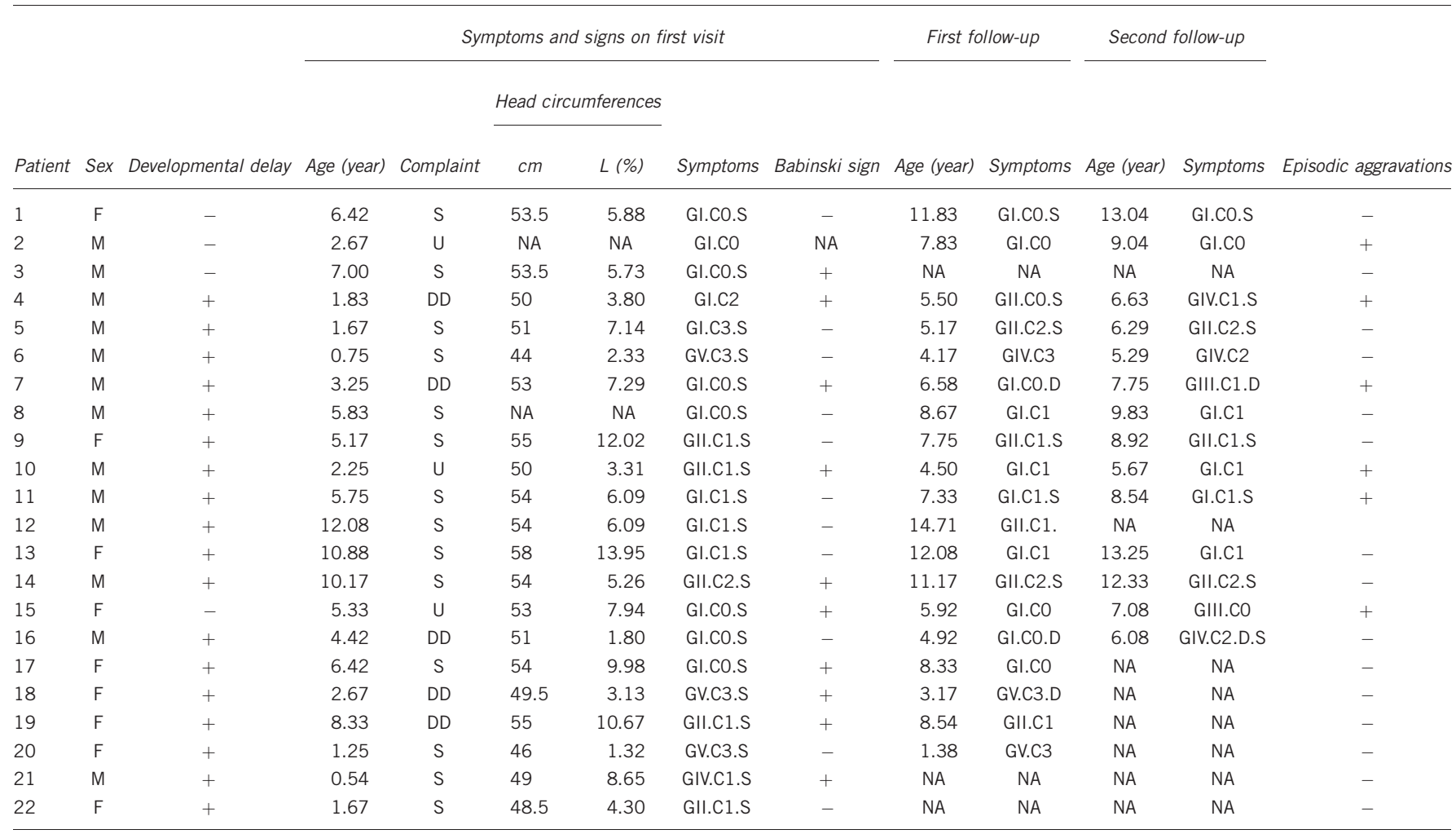

Abbreviations: AxD, Alexander disease; C0-3, cognitive scale; DD, developmental delay; D, dysphagia; F, female; GI-V, GMFSC scale; L, larger than age-matched average; M, male; NA, not available; +, positive; -, negative; $\mathrm{S}$, seizures; $\mathrm{U}$, unable to walk after falling.

At the first follow-up, $7(36.84 \%, n=19)$ showed no cognitive impairment, 7 (36.84\%) were classified as C1, 2 (10.53\%) as C2 and 3 $(15.79 \%)$ as C3. Compared with the first visit, most patients (15, $78.95 \%)$ remained stable, $2(10.53 \%)$ showed regression and 2 (10.53\%) showed improvement. At the second follow-up visit, there were only $3(21.43 \%, n=14)$ without cognitive impairment, 7 were classified as C1 $(50.00 \%)$, and 4 as C2 (28.57\%). Compared with the first follow-up, $10(71.43 \%)$ remained stable, $3(21.43 \%)$ showed decline and $1(7.14 \%)$ showed improvement.

For the 14 cases underwent second follow-up, change in constituent ratio of motor and cognitive scaling showed a gradual increase of patients with functional impairments (Figure 2).
Paroxysmal aggravations and seizures. Paroxysmal aggravations presented in $27.27 \%(n=22)$. These were characterized by acute onset of walking disability and weakness of limbs after infection or trauma, followed by slow recovery. Seizures were present in $95.45 \%$ $(n=22)$ until the last follow-up. They were characterized by partial motor or generalized tonic-clonic seizures. The first seizure occurred at an average of 1.56 years of age (0.33-5.00). Seizures were precipitated by fever in $42.86 \%$. Seizure frequency varied among individuals, from once per month to once per year. Antiepileptic drugs, such as valproate, topiramate or levetiracetam, were prescribed for 16 patients. At the second follow-up, $42.86 \%$ still had seizures. 

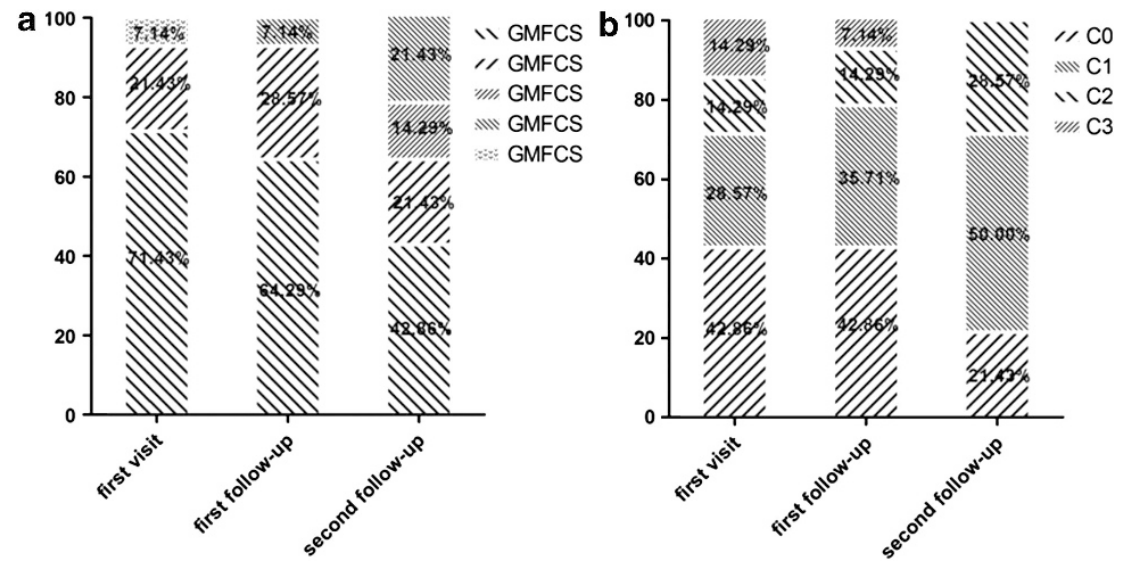

Figure 2 Constituent ratio of motor and cognitive scaling at first visit and follow-up visits. For the 14 cases underwent second follow-up, change in constituent ratio of motor (a) and cognitive scaling (b) showed a gradual increase of patients with functional impairments.

\section{Genotype of patients}

Heterozygous missense mutations of GFAP were detected in 21 $(95.45 \%, n=22)$ (Table 3). Eleven mutations were identified, of which three were novel. Mutations affected p.R79 in $6(28.57 \%$, $n=21)$, p.R239 in $5(23.81 \%)$ and p.R88 in 5 (23.81\%). Mutations were identified in exon 1 in $13(61.91 \%)$, exon 4 in $5(23.81 \%)$, exon 6 in $2(9.52 \%)$ and exon 7 in $1(4.76 \%)$. All mutations were de novo except in patient 17 . The girl inherited the mutation p.R79H from her mother. The mother was 29-years-old with average IQ, and without symptoms of ocular movement abnormalities, bulbar symptoms, seizures or cognitive decline at the time of follow-up. Brain MRI showed abnormalities in deep white matter and atrophy of the medulla oblongata and upper cervical spinal cord (Figure 3).

\section{Parental origin of the allele harbor the de novo mutation}

Allele-specific PCR was performed in 10 cases with available informative single-nucleotide polymorphisms in the $2 \mathrm{~kb}$ region flanking the mutations. In $90 \%$ of cases $(n=10)$, mutations occurred on the paternal allele (Table 1).

\section{DISCUSSION}

\section{Phenotype of $\mathrm{AxD}$}

Modified classification systems of clinical subtype of $\mathrm{AxD}$ have recently been proposed, slightly different from the conventional agedependent system. Yoshida et al. ${ }^{11}$ proposed a guideline to classify $\mathrm{AxD}$ into cerebral (type 1), bulbospinal (type 2) and intermediate (type 3 ) on the basis of neurological and MRI findings. Patients between types 1 and 2, and long-term survivors with infantile $\mathrm{AxD}$ were considered as type 3. A survey in Japan revealed that type $1 \mathrm{AxD}$ accounted for $34.3 \%$ of cases, type 2 accounted for $45.7 \%$ and type 3 accounted for $20 \%$. Based on a latent class analysis on $215 \mathrm{AxD}$ patients to statistically define AxD subtypes, Prust et al. recently proposed to categorize patients as either type I or type II. ${ }^{3,8}$ The modified classification focuses less on the age of onset and more on constellations of symptoms and MRI features. The estimated prevalence rates of types I and II are $60 \%$ and $40 \%$, respectively.

As diagnosis was made on the basis of typical MRI features, all children in our study were classified as type I according to Prust's system. Type I is usually characterized by early onset, macrocephaly, seizures, developmental delay, paroxysmal deterioration and typical MRI findings. The phenotypes of our 22 Chinese patients were consistent with the typical features. Motor development was delayed
Table 3 GFAP mutations in 22 AxD patients

\begin{tabular}{|c|c|c|c|c|c|c|}
\hline Cases & Exon & $\begin{array}{c}\text { Nucleotide } \\
\text { change }\end{array}$ & $\begin{array}{l}\text { Amino-acid } \\
\text { change }\end{array}$ & $\begin{array}{l}\text { Location in pro- } \\
\text { tein domain }\end{array}$ & $\begin{array}{l}\text { Parental } \\
\text { derivation }\end{array}$ & $\begin{array}{l}\text { Novell } \\
\text { reported }\end{array}$ \\
\hline 1 & e1 & c. $262 \mathrm{C}>\mathrm{T}$ & p.R88C & Coil-1A & - & Reported \\
\hline 2 & e1 & c. $262 \mathrm{C}>\mathrm{T}$ & p.R88C & Coil-1A & - & Reported \\
\hline 3 & e1 & c. $262 \mathrm{C}>\mathrm{T}$ & p.R88C & Coil-1A & - & Reported \\
\hline 4 & e4 & c. $715 \mathrm{C}>\mathrm{T}$ & p.R239C & Coil-2A & - & Reported \\
\hline 5 & e4 & c. $715 \mathrm{C}>\mathrm{T}$ & p.R239c & Coil-2A & - & Reported \\
\hline 6 & e7 & c. $1154 \mathrm{C}>\mathrm{T}$ & p.S385F & C-terminal & - & Novel \\
\hline 7 & e6 & c. $1119 \mathrm{G}>\mathrm{C}$ & p.E373D & C-terminal & - & Novel \\
\hline 8 & e1 & c. $236 \mathrm{G}>\mathrm{T}$ & p.R79L & Coil-1A & - & Reported \\
\hline 9 & e1 & c. $226 C>T$ & p.L76F & Coil-1A & - & Reported \\
\hline 10 & e1 & c. $262 \mathrm{C}>\mathrm{A}$ & p.R88S & Coil-1A & - & Reported \\
\hline 11 & e1 & c. $262 \mathrm{C}>\mathrm{T}$ & p.R88C & Coil-1A & - & Reported \\
\hline 12 & - & - & - & - & - & - \\
\hline 13 & e1 & c. $236 \mathrm{G}>\mathrm{A}$ & p.R79H & Coil-1A & - & Reported \\
\hline 14 & e1 & c. $235 \mathrm{C}>\mathrm{T}$ & p.R79C & Coil-1A & - & Reported \\
\hline 15 & e1 & c. $231 \mathrm{~T}>\mathrm{A}$ & p.N77K & Coil-1A & - & Novel \\
\hline 16 & e1 & c. $235 \mathrm{C}>\mathrm{T}$ & p.R79C & Coil-1A & - & Reported \\
\hline 17 & e1 & c. $236 \mathrm{G}>\mathrm{A}$ & p.R79H & Coil-1A & Maternal & Reported \\
\hline 18 & e4 & c. $716 \mathrm{G}>\mathrm{A}$ & p.R239H & Coil-2A & - & Reported \\
\hline 19 & e1 & c. $235 \mathrm{C}>\mathrm{T}$ & p.R79C & Coil-1A & - & Reported \\
\hline 20 & e4 & c. $716 \mathrm{G}>\mathrm{A}$ & p.R239H & Coil-2A & - & Reported \\
\hline 21 & e4 & c. $716 \mathrm{G}>\mathrm{A}$ & p.R239H & Coil-2A & - & Reported \\
\hline 22 & e6 & c. $1120 \mathrm{G}>\mathrm{C}$ & p.E374Q & C-terminal & - & Reported \\
\hline
\end{tabular}

Abbreviations: AxD, Alexander disease; GFAP, glial fibrillary acidic protein; -, negative.

in $81.82 \%$, and cognitive delay in $63.64 \%$. Macrocephaly was noticed in most patients. Seizures have been reported in $92 \%$ of infantile patients, $39.1 \%$ with juvenile $\mathrm{AxD}$ and $16.1 \%$ with adult $\mathrm{AxD} .^{1,12}$ In our study, $95.45 \%$ experienced recurrent seizures, with $42.86 \%$ precipitated by fever. The first seizure occurred at as early as 4 months of age, with an average of 1.56 years. The earlier onset and higher prevalence of seizures in type I AxD is probably due to the early involvement of subcortical white matter. Paroxysmal deterioration was more common in type I than in type II. ${ }^{3}$ Episodic aggravations presented in $27.27 \%$ of our patients. Paroxysmal deterioration after febrile illness or head trauma has been reported in other hereditary leukoencephalopathies, especially in vanishing white matter disease, which is supposed to be related to endoplasmic reticulum (ER) stress in glial cells. ${ }^{13,14}$ Disease progression is 


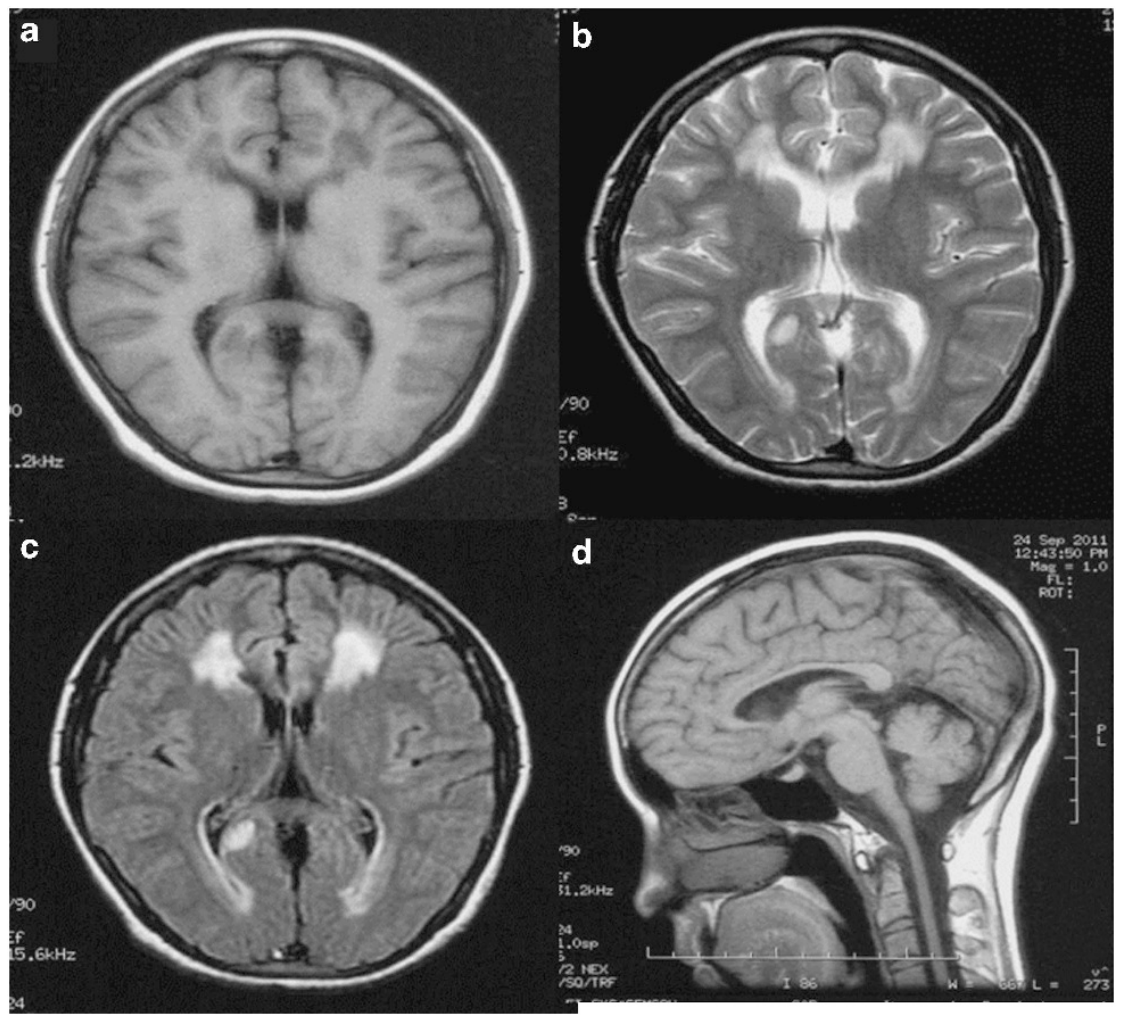

Figure 3 The brain magnetic resonance imaging of the presymptomatic mother with type II AxD. (a) (T1 image), (b) (T2 image) and (c) (Flair image) showed abnormalities in periventricular white matter, and atrophy of the medulla oblongata and upper cervical spinal cord (d).

much more rapid in type I than in type II. The survival analysis in Prust's study revealed a mean survival of $14.0 \pm 1.8$ years in type I. At 8.55 years of age (5.29-13.25), all patients who underwent the second follow-up in our study were alive. The motor or cognitive function was slowly gradually impaired in most patients, with GMFCS I still preserved in $42.86 \%$, and C0 in $21.43 \%$. Further follow-up of this cohort is needed to fully understand the progressive process in Chinese patients.

Type II AxD is characterized by late onset, autonomic dysfunction, ocular movement abnormalities, bulbar symptoms, palatal myoclonus and atypical MRI findings. Signal abnormalities and/or atrophy of the medulla oblongata and cervical spinal cord are common features on MRI. ${ }^{15,16}$ Some atypical imaging features were reported, like focal lesions, abnormal signals in cerebellum and calcifying lesions in the sub-cortex and cortex. ${ }^{17-19}$ The survival analysis in Prust's study revealed a mean survival of $25.0 \pm 2.1$ years. It was reported that half of the late-onset cases were familial, whereas familial cases are very rare in infantile type. ${ }^{20,21}$ In our study, the mother of patient 17 was demonstrated to have a heterozygous GFAP mutation p.R79H. She was 29-years-old without any symptoms at the time of the follow-up. Brain MRI showed common features of Type II AxD. Given the $100 \%$ penetrance of GFAP mutations, she should be a presymptomatic patient. The age of disease onset in type II $\mathrm{AxD}$ is estimated to be $21.64 \pm 2.35$ years, ${ }^{3}$ with a maximum age of 63 years. ${ }^{1}$ Follow-up of the mother is needed. To our knowledge, this is the second case of late-onset $\mathrm{AxD}$ with a p.R79H mutation worldwide.

\section{Genotype of AxD}

GFAP encodes glial fibrillary acidic protein. It is an astrocytic intermediate filament that forms the cytoskeleton together with microfilaments and microtubules, with participates in cytoskeleton restructuring, cell adhesion and myelination. ${ }^{22}$ It is composed of a central $\alpha$-helical rod domain flanked by a non- $\alpha$-helical $N$-terminal head and $\mathrm{C}$-terminal tail domains. The rod domain comprises four helical domains (1A, 1B, 2A and 2B), separated by short linkers (L1, L12 and L2). ${ }^{23}$ Possible pathogenic mechanisms underlying AxD include GFAP aggregation, oxidative stress, autophagic response in glia and decreased transfer of glutamine from astrocytes to neurons. $^{24-26}$

GFAP sequencing can identify mutations in $>90 \%$ of $\mathrm{AxD}$ patients. To date, almost 100 different mutations have been reported. ${ }^{11}$ More than half involved in one of the hot spots including p.R239 (20.3\%), p.R79 (16.6\%), p.R88 (7.9\%) and p. R416 (5.6\%). ${ }^{3}$ In our study, mutations affected p.R79 in $28.57 \%$, p.R239 in $23.81 \%$ and p.R88 in $23.81 \%$, with a higher prevalence of p.R88 mutations. All mutations identified in our study are missense, consistent with other reports. Small deletions and short in-frame insertions have been found in a few patients. ${ }^{8}$ It was reported that GFAP mutations were identified in exon 1 in $45.2 \%$ of cases, exon 3 in $3.3 \%$, exon 4 in $27.2 \%$, exon 5 in $1.8 \%$, exon 6 in $16.0 \%$, exon 7 in $<1 \%$ and exon 8 in $7.5 \%$. No mutations were found in exons 2 and $9 .{ }^{3}$ In our study, mutations were identified in exon $1(61.90 \%)$, exon $4(23.81 \%)$, exon $6(9.52 \%)$, and exon7 $(4.76 \%)$. The distribution of mutations across the GFAP domains was reported as follows: $\mathrm{N}$-terminal head $(<1 \%$ of cases), coil-1A $(43.7 \%)$, coil $1 \mathrm{~B}(4.2 \%)$, L12 $(<1 \%)$, coil-2A (23.7\%), L2 $(<1 \%)$, coil $2 \mathrm{~B}(13.0 \%)$, and C-terminal tail (13.5\%). No mutations have been identified in the L1 region. ${ }^{3}$ The mutations identified in our cases were distributed in coil-1A (61.90\%), coil-2A (23.80\%), and C-terminal tail (14.29\%). We could not identify a GFAP mutation by sequencing in one patient. 
Mutations in untranslated regions or deep introns, or copy number variations (especially duplication of GFAP), probably underlie the disease in nearly $10 \%$ of mutation-negative patients.

\section{Genotype-phenotype correlation}

It was reported that type I shows higher incidence of p.R79 and p.R239 mutations than type II. $^{3}$ A few studies analyzed the phenotypic difference between mutations in p.R79 and p.R239. Patients with p.R239 mutations appear to be more severe. ${ }^{1,27,28}$ Like other inherited disorders, the genotype of GFAP is absolutely not the only determinant factor for phenotype. Patient 17 and her mother, who shared the same genotype, showed totally different phenotype. Therefore, other genetic or environmental factors influence the phenotype.

\section{Tendency for de novo GFAP mutation on the paternal allele} In $90 \%$ of analyzed cases in our study, the de novo mutations were harbored on the paternal allele. This result is consistent with a previous research, which showed that de novo GFAP mutations were on the paternal allele in $85.7 \%$ of cases $(n=28) .{ }^{29}$ It is possible that GFAP mutations have a high frequency of male germ-line transmission. De novo mutations tend to occur on the paternal allele in other genetic neurological diseases, like Rett syndrome and Dravet syndrome. ${ }^{30,31}$ There are several potential explanations for this male-biased mutation. Most point mutations at a $\mathrm{CpG}$ nucleotide pair are caused by methylation. ${ }^{32}$ Methylation occurs more during spermatogenesis. ${ }^{33}$ This may explain why $43.6 \%$ of GFAP mutations occurred at CpG methylation sites. ${ }^{11}$ More cell divisions in the life history of sperm also increase the possibility of errors. ${ }^{34}$

\section{CONCLUSIONS}

This is the largest follow-up study on Chinese children with type I AxD. The phenotypes of these patients are consistent with reports in other populations. The mother of a proband was demonstrated to be a presymptomatic patient with type II $\mathrm{AxD}$, who is the second case of late-onset $\mathrm{AxD}$ with a p.R79H mutation worldwide. Reported hot spot mutations of GFAP, p.R79, p.R239 and p.R88, were also identified in Chinese patients. Our data showed that $90 \%$ of de novo GFAP mutations were on the paternal allele, suggesting male germline transmission. More patients and a longer follow-up period are needed for a better understanding of the clinical and genetic characteristics of $\mathrm{AxD}$.

\section{ACKNOWLEDGEMENTS}

The study was supported by the program for new century excellent talents in university from the Chinese Ministry of Education and Natural Science Foundation of China (81171065).

1 Li, R., Jonhson, A. B., Salomons, G., Goldman, J. E., Naidu, S., Quinlan, R. \& Wu, Y Glial fibrillary acidic protein mutations in infantile, juvenile, and adult forms of Alexander disease. Ann. Neurol. 57, 310-326 (2005).

2 Johnson, A. B. \& Brenner, M. Alexander disease: a review and the gene. Int. J. Dev. Neurosci. 20, 391-394 (2002).

3 Prust, M., Wang, J., Morizono, H., Messing, A., Brenner, M., Gordon, E. et al. GFAP mutations, age at onset, and clinical subtypes in Alexander disease. Neurology 77, 1287-1294 (2011)

4 Johnson, A. B. \& Bettica, A. On-grid immunogold labeling of glial intermediate filaments in epoxy-embedded tissue. Am. J. Anat. 185, 335-341 (1989).

5 Head, M. W., Corbin, E. \& Goldman, J. E. Overexpression and abnormal modification of the stress proteins alpha B-crystallin and HSP27 in Alexander disease. Am. J. Pathol. 143, 1743-1753 (1993)
6 Brenner, M., Johnson, A. B., Boespflug-Tanguy, O., Rodriquez, D., Goldman, J. E. \& Messing, A. Mutations in GFAP, encoding glial fibrillary acidic protein, are associated with Alexander disease. Nat. Genet. 27, 117-120 (2001).

7 Brenner, M., Goldman, J. E., Quinlan, R. A. \& Messing, A. Alexander disease: a genetic disorder of astrocytes. In: Astrocytes in (Patho) Physiology of the Nervous System (eds Parpura, V. \& Haydon, P. G.) pp 591-648 (Springer, New York, 2009).

8 Messing, A., Daniels, C. M. \& Hagemann, T. L. Strategies for treatment in Alexander disease. Neurotherapeutics 7, 507-515 (2010).

9 van der Knaap, M. S., Naidu, S., Breiter, S. N., Blaser, S., Stroink, H., Springer, S. et al. Alexander disease: diagnosis with MR imaging. Am. J. Neuroradiol. 22, 541-552 (2001).

10 Shi, W., Wang, S. J., Yang, H., Zhu, M. \& Wang, L. Study on reliability and validity of the Chinese version of the gross motor function classification system for cerebral palsy. Chin. J. Evld. Based Pediatr. 1, 122-129 (2006).

11 Yoshida, T. \& Nakagawa, M. Clinical aspects and pathology of Alexander disease, and morphological and functional alterationof astrocytes induced by GFAP mutation. Neuropathlogogy doi:10.1111/j.1440-1789 (2011).

12 Balbi, P., Salvini, S., Fundarò, C., Frazzitta, G., Maestri, R., Mosah, D. et al. The clinical spectrum of late-onset Alexander disease: a systematic literature review. J. Neurol. 257, 1955-1962 (2010).

13 van der Knaap, M. S., Pronk, J. C. \& Scheper, G. C. Vanishingwhite matter disease. Lancet Neurol. 5, 413-423 (2006).

14 D’Antonio, M., Feltri, M. L. \& Wrabetz, L. Myelin under stress. J. Neurosci. Res. 87, 3241-3249 (2009).

15 Probst, E. N., Hagel, C., Weisz, V., Nagel, S., Wittkugel, O., Zeumer, H. et al. Atypical focal MRI lesions in a case of juvenile Alexander's disease. Ann. Neurol. 53, 118-120 (2003).

16 van der Knaap, M. S., Salomons, G. S., Li, R., Franzoni, E., Gutiérrez-Solana, L. G., Smit, L. M. et al. Unusual variants of Alexander's disease. Ann. Neurol. 57, 327-338 (2005).

17 Barreau, P., Prust, M. J., Crane, J., Loewenstein, J., Kadom, N. \& Vanderver, A. Focal central white matter lesions in Alexander disease. J. Child. Neurol. 26, 1422-1424 (2011).

18 Schmidt, S., Wattjes, M. P., Gerding, W. M. \& van der Knaap, M. Late onset Alexander's disease presenting as cerebellar ataxia associated with a novel mutation in the GFAP gene. J. Neurol. 258, 938-940 (2011).

19 Jefferson, R. J., Absoud, M., Jain, R., Livingston, J. H., Van der Knaap, M. S. \& Jayawant, S. Alexander disease with perivent ricular calcification: a novel mutation of the GFAP gene. Dev. Med. Child. Neurol. 52, 1160-1163 (2010).

20 Namekawa, M., Takiyama, Y., Honda, J., Shimazaki, H., Sakoe, K. \& Nakano, I. Adultonset Alexander disease with typical " tadpole" brainstem atrophy and unusual bilateral basal ganglia involvement: a case report and review of the literature. BMC Neurol. 10, 21 (2010).

21 Johnson, A. B. \& Brenner, M. Alexander's disease: clinical, pathologic, and genetic features. J. Child. Neurol. 18, 625-632 (2003)

22 Eng, L. F., Ghirnikar, R. S. \& Lee, Y. L. Glial fibrillary acidic protein: GFAP-Thirty-One Years (1969-2000). Neurochem. Res. 25, 1439-1451 (2000).

23 Sawaishi, Y. Review of Alexander disease: beyond the classical concept of leukodystrophy. Brain Dev. 31, 493-498 (2009).

24 Wang, L., Colodner, K. J. \& Feany, M. B. Protein misfolding and oxidative stress promote glial-mediated neurodegeneration in an Alexander disease model. J. Neurosci. 31, 2868-2877 (2011).

25 Meisingset, T. W., Risa, Ø., Brenner, M., Messing, A. \& Sonnewald, U. Alteration of glial-neuronal metabolic interactions in a mouse model of Alexander disease. Glia $\mathbf{5 8}$ 1228-1234 (2010).

26 Messing, A., Brenner, M., Feany, M. B., Feany, M. B., Nedergaard, M. \& Goldman, J. E. Alexander disease. J. Neurosci. 32, 5017-5023 (2012).

27 Rodriguez, D., Gauthier, F., Bertini, E., Bugiani, M., Brenner, M., N'guyen, S. et al. Infantile Alexander disease: spectrum of GFAP mutations and genotype-phenotype correlation. Am. J. Hum. Genet. 69, 1134-1140 (2001).

28 Brenner, M., Johnson, A. B., Boespflug-Tanguy, O., Rodriguez, D., Glodman, J. E. \& Messing, A. Mutations in GFAP, encoding glial fibrillary acidic protein, are associated with Alexander disease. Nature Genet. 27, 117-120 (2001).

29 Li, R., Jonhson, A. B., Salomons, G. S., van der Knaap, M. S., Rodriguez, D., Boespflug-Tanguy, O. et al. Propensity for paternal inheritance of de novo mutations in Alexander disease. Hum. Genet. 119, 137-144 (2006).

30 Girard, M., Couvert, P., Carrié, A., Tardieu, M., Chelly, J., Beldjord, C. et al. Parental origin of de novo MECP2 mutations in Rett syndrome. Eur. J. Hum. Genet. 9, 231-236 (2001).

31 Heron, S. E., Scheffer, I. E., Iona, X., Zuberi, S. M., Birch, R., McMahon, J. M. et al. De novo SCN1A mutations in Dravet syndrome and related epileptic encephalopathies are largely of paternal origin. J. Med. Genet. 47, 137-141 (2010).

32 Robertson, K. D. \& Wolffe, A. P. DNA methylation in health and disease. Nat. Rev Genet. 1, 11-19 (2000)

33 Monk, M. Epigenetic programming of differential gene expression in development and evolution. Dev. Genet. 17, 188-197 (1995).

34 Taylor, J., Tyekucheva, S., Zody, M., Chiaromonte, F. \& Makove, K. D. Strong and weak male mutation bias at different sites in the primate genomes: insights from the humanchimpanzee comparison. Mol. Biol. Evol. 23, 565-573 (2006). 